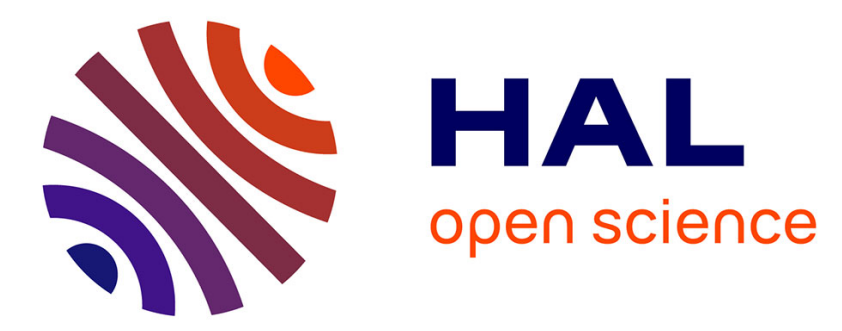

\title{
Indentation for estimating the human tongue soft tissues constitutive law: application to a 3D biomechanical model
}

Jean-Michel Gérard, Jacques Ohayon, Vincent Luboz, Pascal Perrier, Yohan Payan

\section{To cite this version:}

Jean-Michel Gérard, Jacques Ohayon, Vincent Luboz, Pascal Perrier, Yohan Payan. Indentation for estimating the human tongue soft tissues constitutive law: application to a 3D biomechanical model. Lecture Notes in Computer Science, 2004, 3078, pp.77-83. hal-00081929

\section{HAL Id: hal-00081929 \\ https://hal.science/hal-00081929}

Submitted on 26 Jun 2006

HAL is a multi-disciplinary open access archive for the deposit and dissemination of scientific research documents, whether they are published or not. The documents may come from teaching and research institutions in France or abroad, or from public or private research centers.
L'archive ouverte pluridisciplinaire HAL, est destinée au dépôt et à la diffusion de documents scientifiques de niveau recherche, publiés ou non, émanant des établissements d'enseignement et de recherche français ou étrangers, des laboratoires publics ou privés. 


\title{
Indentation for estimating the human tongue soft tissues constitutive law: application to a 3D biomechanical model
}

\author{
J.M. Gérard ${ }^{1,2}$, J. Ohayon ${ }^{2}$, V. Luboz ${ }^{2}$, P. Perrier ${ }^{1}$, Y.Payan ${ }^{2}$ \\ ${ }^{1}$ Institut de la Communication Parlée, UMR CNRS 5009, INPG, Grenoble, France \\ \{gerard, Pascal.Perrier \} @icp.inpg.fr \\ ${ }^{2}$ Laboratoire TIMC, CNRS, Université Joseph Fourier, Grenoble, France \\ \{Jacques.Ohayon, Vincent.Luboz, Yohan.Payan\} @imag.fr
}

\begin{abstract}
A 3D biomechanical model of the tongue is presented here. Its goal is to evaluate the speech control model. This model was designed considering three constraints: speech movement speed, tongue movements and tongue soft tissue mechanical properties. A model of the tongue has thus been introduced taking into account the non linear biomechanical behavior of its soft tissues in a large deformation analysis. Preliminary results showed that the finite element model is able to simulate the main movements of the tongue during speech data. It seems that the model may be used in the estimation of glossectomy impacts on patient speak and on different surgery approaches.
\end{abstract}

\section{Introduction}

The A 3D biomechanical model of the tongue is developed to serve as a tool for future evaluations of speech motor control models. This model was designed considering three constraints, crucial for speech tasks. Firstly, speech movements are rapid (around 50ms) and their time variations determine important cues for speech perception: the model must therefore handle dynamics [1] and be able to produce fast gestures. Secondly, tongue movements are resulting from the interaction between muscle commands, muscle anatomy, tongue tissues mechanical properties and external factors such as the teeth, palate or pharyngeal walls. An accurate representation of tongue muscular structure, a complete description of vocal tract and a realistic account of tongue elasticity are therefore required to design the model. Tongue soft tissues feature non linear mechanical properties, mostly due to muscle fibers interweaving. Moreover, as reported in [2], the relative tongue deformations can reach a rate of $200 \%$ in compression. The model has then to handle non linear mechanical properties and to assume a large deformation framework. Model design and simulations were carried out with the use of the Finite Element package ANSYS ${ }^{\mathrm{TM}}$.

A basic obstacle encountered in the design of such a model arises from the lack of experimental data giving quantitative information about the mechanical properties of tongue tissues. This is the motivation of this paper, in which the tongue model will be first briefly described before the experimental and computational procedure will be 
presented that we have set up to quantitatively measure the elastic properties of a human tongue.

\section{Design of the Mesh}

The Finite Element Method [3] was used for the discretization of the equations of motion. This method requires the design of a mesh, based on biological data providing an accurate description of the tongue. The Visible Human Project ${ }^{\mathbb{R}}$, s female data set, in parallel with morphological studies of tongue musculature, was used to create the original mesh (Figure 1). This process is described in details in [4] while the definition of internal muscular structures is explained in [5].

In order to facilitate further quantitative comparisons of simulations with experimental data collected on real speakers, the external geometry of the initial mesh was matched to the 3D tongue shape of a human speaker tongue shape, while preserving its internal structure at rest. Reference data consisted in the association of MRI images, CT scans (for bony structures like jaw and hyoid bone), and plaster cast of the palate optically scanned to digitize the information.
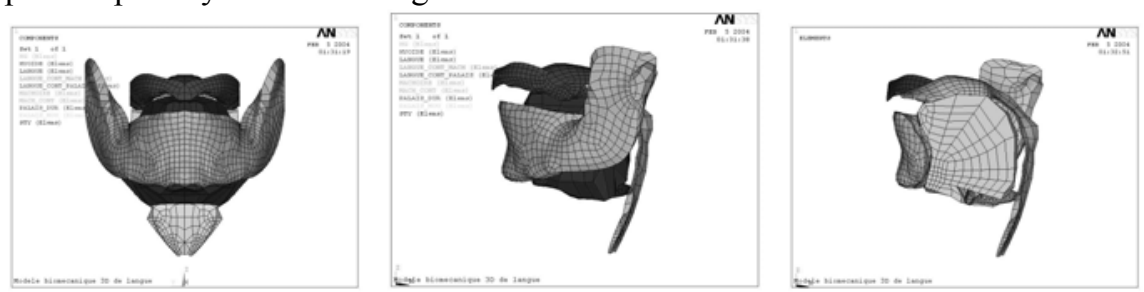

Fig. 1. 3D Finite Element mesh (Front view, Side view, Sagittal view)

\section{Mechanical Modelling}

\subsection{Simulation Framework and Choice of the Material}

Tongue models previously published by [6], [7], [8], [9], and [10] were based on small deformation framework hypothesis. However, [2] reported that tongue deformation can reach a rate of $200 \%$ in compression and $160 \%$ in elongation during speech tasks, which is totally out of the small deformation framework. This is why a large deformations framework was assumed here.

Biological soft tissues can feature non linear, anisotropic and viscoelastic mechanical properties [11]. The non linearities are accounted in the model, using hyper elastic material. A material is said to be hyper elastic if we can find an energy function $\mathrm{W}$ so that its derivative respect to the strain $\mathrm{E}$ equals the stress tensor $\mathrm{S}$ : 


$$
S=\frac{\partial W}{\partial E} \quad E=\frac{1}{2}\left(F^{T} F-I\right)
$$

$\mathrm{E}$ is the lagrangian deformation tensor, $\mathrm{F}$ is deformation gradient and $\mathrm{I}$ is the identity matrix.

For our model, the energy function $\mathrm{W}$ was approximated by a 5 coefficients MooneyRivlin material, so that:

$$
W=a_{10}\left(I_{1}-3\right)+a_{20}\left(I_{1}-3\right)^{2}+a_{01}\left(I_{2}-3\right)+a_{02}\left(I_{2}-3\right)^{2}+a_{11}\left(I_{1}-3\right)\left(I_{2}-3\right) \text {. }
$$

where I1 and I2 are the first and the second invariant of the deformation tensor E. In a first approximation, the model was assumed to be isotropic.

\subsection{Evaluation of Tongue Mechanical Properties}

An indentation experiment was provided on a fresh human cadaver tongue (Figure 2, left). The indenter is a $14 \mathrm{~mm}$ radius cylinder, applying increasing loads locally on the tongue. The vertical displacement of the indenter is measured as a function of the applied load.
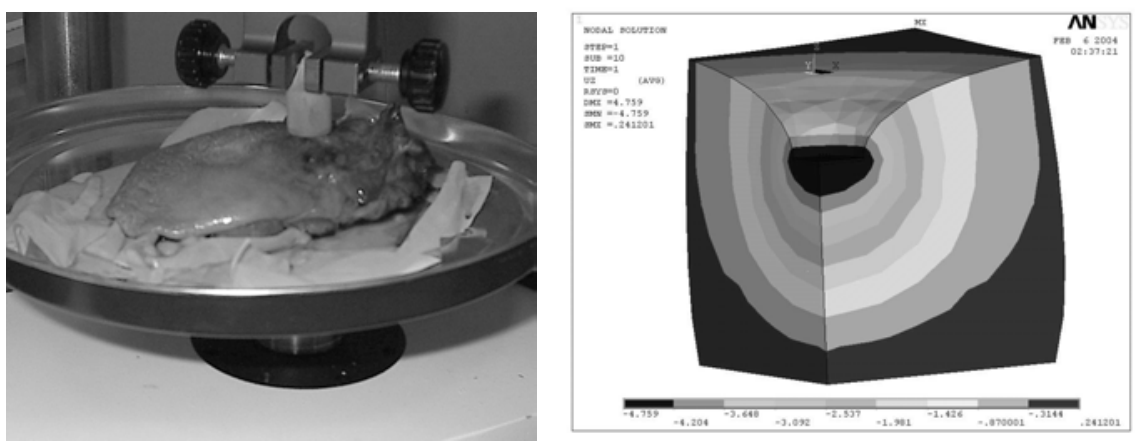

Fig. 2. Experimental indenter (left) and its numerical model (right)

Unfortunately, these data can't provide direct results for the constitutive law of the material. In order to get to the stress/strain relationship (i.e. the Mooney-Rivlin coefficients) from them, the indentation experiment was numerically simulated, with a finite element model of the lingual tissues laid on a table and in contact with an indenter (note that figure 2 only plots a quarter of the simulation: axisymetric assumption). A first series of 5 experiments was done in the front part of the tongue, and another series was done in the rear. Loads are gradually applied from 0 to $1 \mathrm{~N}$, with $0.1 \mathrm{~N}$ steps. During relaxation, loads were applied from $1 \mathrm{~N}$ to $0 \mathrm{~N}$ with $0.2 \mathrm{~N}$ steps (Figure 3). 


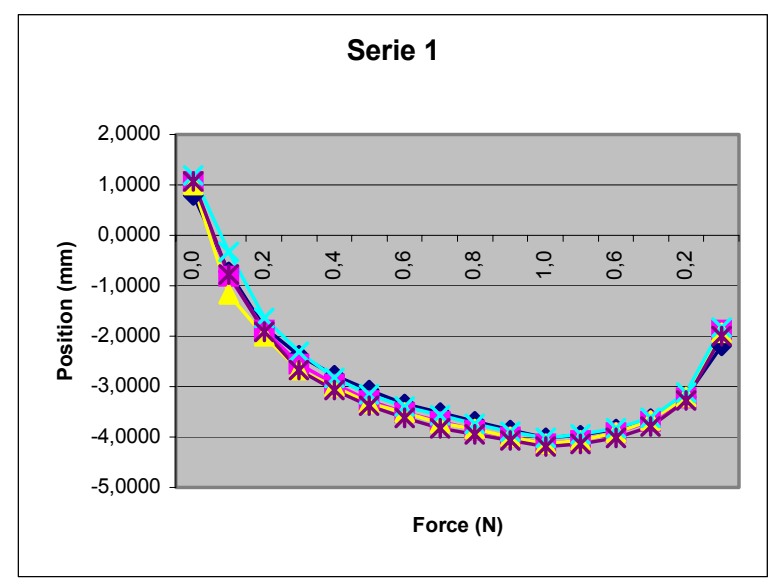

Fig. 3. Example of results for a set of 5 experiments (Load experiment)

It should be noted that, due to tongue plasticity, tongue shape after relaxation is not the same as initial tongue shape. Thus, after each relaxation, the tongue was manually reshaped so that initial conditions can be supposed to be essentially the same for each experiment. The mucosa covering the surface of the tongue has mechanical properties different from the muscles. Hence, two additional series of measurements were carried out after removing the mucosa. In summary, two series of experiments provide data on muscular tissues only whereas the two other series provide data including the mucosa.

Then, the determination of the constitutive law was done in two steps. A first model of the numerical indenter with a single layer structure was designed to compute the Mooney-Rivlin coefficients for the anterior and posterior parts of the tongue separately. In a second step and given the coefficients for muscle tissue computed previously, the mucosa constitutive law was computed, once for the front of the tongue and once for the rear. Then an iterative process was used to find the Mooney-Rivlin coefficients so that simulated displacements fit with experimental displacements. For each of the four sets of experiments, one constitutive law was computed and the Mooney-Rivlin coefficients were calculated using the mean of the 5 displacement/force measured. An iterative algorithm, based on a dichotomic convergence process, was designed to compute these coefficients.

\subsection{Boundary conditions and equation solving}

The model is assumed to be incompressible. The Poisson ratio í should then be as close as possible to a 0.5 value. However, increasing the í value dramatically increases computation time. Consequently, different values of í were tested, ending up by choosing a 0.49 value for í. This value corresponds to a volume variation lower than $2 \%$ with reasonable computation times. The gravity value was fixed to $\mathrm{g}=9.81 \mathrm{~m} . \mathrm{s}-2$ and the density to $1000 \mathrm{~kg} / \mathrm{m} 3$. The corresponding mass value was therefore around $140 \mathrm{~g}$. To take into account the visco-elasticity of tongue tissues, the 
Rayleigh damping model was assumed, and damping coefficients were chosen to reach critical damping.

The forces are applied along macro-fibers defined by a series of nodes along the edges of the elements. Forces are mainly concentrated at the ends of fibers and aim at reducing the fiber length when the muscle is activated. However, the fibers are initially curved and, in order to account for the fact that muscular activation tends to straighten fibers, forces depending on the fibers curvature are distributed along the fibers (cf. [10])

These equations are solved by the FE package ANSYSTM which computes the constraints, the deformations and displacements at each node of the mesh.

\section{Results}

Figure 4 plots the experimental results and the estimated constitutive law for the rear part of the tongue without mucosa. The dots are experimental measurements and the curve represents the constitutive law $(\mathrm{a} 10=0.42 \mathrm{kPa}$ and $\mathrm{a} 20=12.5 \mathrm{kPa}$ ). The maximum gap between experimental and simulated data is $0.22 \mathrm{~mm}$.

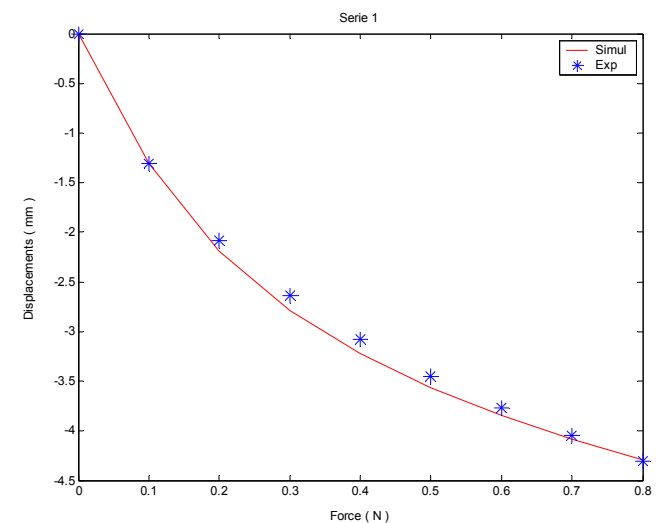

Fig. 4. Experimental results and estimated constitutive law for the rear of the tongue without mucosa

Once the constitutive law of tongue soft tissues was calculated, it was implemented in the biomechanical tongue model. The model was then tested with force levels compatible with speech movements. Here is an example of deformations obtained by specific muscle activation. The duration of each simulation is $120 \mathrm{~ms}$ and the forces are applied as a step function for all simulations. Tongue shapes in the mid-sagittal plane, at rest and at the end of the simulation, are shown in Fig 5 for the simultaneous activations of the Posterior Genioglossus $(6 \mathrm{~N})$, and the Transversalis muscle $(2 \mathrm{~N})$. Expected results are a back to front movement of the tongue with an elevation of tongue dorsum toward the palate. Our simulations are in agreement with these predictions. 

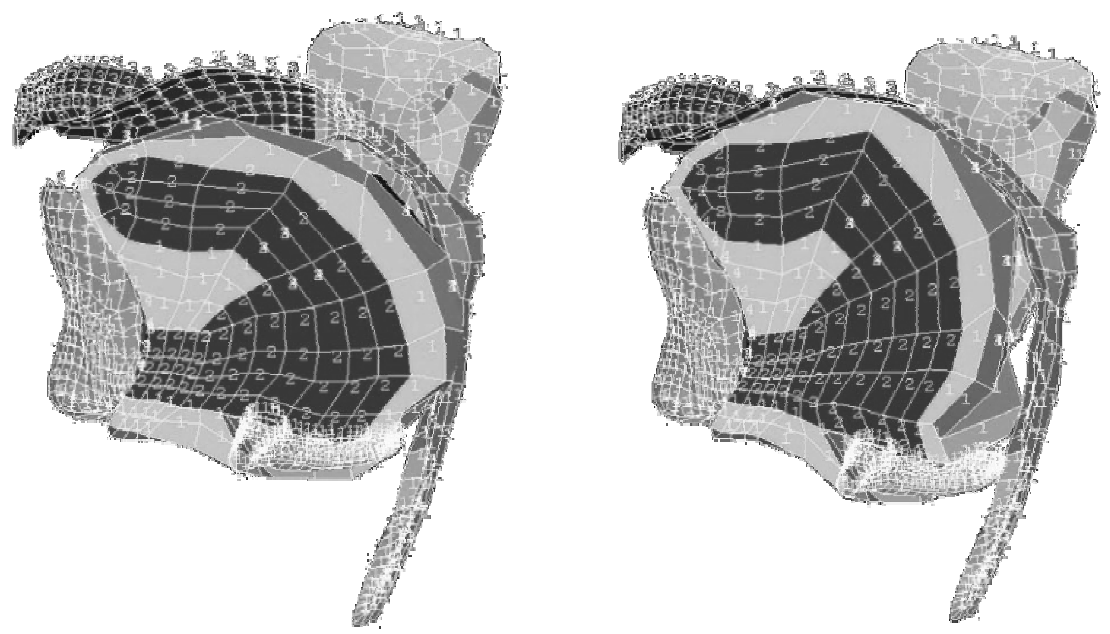

Fig 5. Model in rest position (left) and final position (right) obtained with parallel activation of Genioglossus posterior $(6 \mathrm{~N})$ and Transversalis $(2 \mathrm{~N})$

\section{Conclusion and perspectives}

Preliminary studies on each muscle activation on tongue shape show that main trends of deformations are handled by this model within durations and with level of forces that are fully compatible with speech data.

Impact of each muscle on tongue shape will be evaluated more precisely in future works. This model will be evaluated by comparison of our simulations with measurements of the shape of the vocal tract in the mediosagittal plan. The impacts of these muscle activations are compared to MRI measurements of tongue shapes for a selected number of sounds, for which EMG activations were published in the literature.

This biomechanical tongue model is to be used for pathological applications as a tool to help surgeons planning medical gestures during glossectomy or sleep apnea surgery. In both cases, this model could be used to estimate the impact of surgery gestures on patient's speaking abilities, and if necessary help surgeons to plan a different surgery strategy, less disturbing in terms of speech production abilities.

\section{References}

1. Sanguinetti V., Laboissière R, Ostry D.J. (1998), A dynamic biomechanical model for the neural control of speech production. JASA, Vol 103, pp 1615-1627

2. Napadow V.J., Chen Q., Wedeen V.J. and Gilgert R.J. (1999). Journal of Biomechanics, 32, $1-12$. 
3. Zienkiewicz O.C. and Taylor R.L., 1994. The Finite Element Method, fourth edition. McGraw-Hill Book Company

4. Wilhelms-Tricarico R. (2000). 5th Speech Production Seminar (pp. 141-144). Seeon Bavaria

5. Gérard J.M, Wilhelms-Tricarico R., Perrier P., Payan (2003), Y, A 3D dynamical biomechanical tongue model to study speech motor control, Recent Research and Developments in Biomechanics, Vol 1, pp 49-64

6. Kiritani S., Miyawaki K. and Fujimura O., (1976), A computational model of the tongue. Annual report of the research institute of logopedics and phoniatrics, 10, 243-252, Tokyo University

7. Kakita Y.; Fujimura O., and Honda K., (1985), Computation of mapping from muscular contraction patterns to formant patterns in vowel space, V.A. Fromkin (Ed.), Phonetic and Linguistics (pp. 133-144), Orlando, Florida: Academic press

8. Hashimoto K., Suga S. (1986), Estimation of the muscular tensions of the human tongue by using a threedimensional model of the tongue, Journal of Acoustical society of Japan (E), 7(1),39-46

9. Honda K. (1996), The organisation of tongue articulation for vowels, Journal of Phonetics, 24(1),39-52

10. Y.C. Fung. Biomechanics : Mechanical properties of living tissues. New-York, SpringerVerlag, 1993

11. P.Perrier, Y. Payan, M. Zandipour et J. Perkell. Influences of tongue biomechanics on speech movements during the production of velar stop consonants: A modeling study. JASA, Volume 114, Issue 3, pages 1582-1599, 2003 